\title{
Granular Superconductivity: a Playground for Josephson, Anderson, Kondo, and Mott
}

\author{
Guy Deutscher ${ }^{1}$
}

Published online: 3 February 2021

(C) The Author(s), under exclusive licence to Springer Science+Business Media, LLC part of Springer Nature 2021

\section{Discovery of Granular Superconductivity at the Princeton RCA Labs}

Granular superconductors were discovered accidentally by the group of Ben Abeles at the Princeton RCA Laboratories in the 1960s [1]. They found out that aluminum thin films produced by thermal evaporation in a vacuum system could become superconducting at a temperature of about $2 \mathrm{~K}$, substantially higher than that of bulk aluminum, $1.2 \mathrm{~K}$. At the same time, the normal state resistivity increased up to values several orders of magnitude higher than that of clean aluminum, and their critical field could also be much higher than that of pure aluminum, reaching values of the order of several Tesla.

It turned out that these interesting properties were the result of a poor vacuum. Transmission Electron Microscopy (TEM) pictures of films revealed a granular structure. But when evaporated under a vapor pressure of less the $1.10^{-6}$ Torr, the granular structure disappeared and films just behaved as regular aluminum. Abeles and his colleagues reached the conclusion that granularity was the result of the formation of thin aluminum oxide barriers formed by reaction with oxygen in the vacuum chamber and that these barriers separated aluminum grains. Roger Cohen argued that these barriers reduced the Pippard coherence length and turned aluminum into a type II superconductor [2]. As long as the coherence length was larger than several grains, the details of the granular structure where in fact irrelevant, as Abrikosov vortices could form in the same way as they do in homogeneous type II superconductors were the coherence length is reduced by impurity scattering on the atomic scale. As for the higher critical temperature, Parmenter argued that it was the result of peaks in the

Guy Deutscher

guyde@post.tau.ac.il

1 School of Physics and Astronomy, Tel Aviv University, Ramat Aviv, 69879 Tel Aviv, Israel density of states due to electron confinement in the small grains [3]. In a sense, everything was understood.

\subsection{The Tel Aviv-Rutgers Collaboration}

I had visited Ben Abeles and his group in 1968 while I was a post-doc at Rutgers in the group of Peter Lindenfeld. I was much interested in their results. However, it seemed to me that further progress was necessary. Back at Orsay after my postdoc, I had realized that strong fluctuation effects should be taken into account. In particular, the precise size of the aluminum grains could be of importance, as below a certain critical size of the order of $5 \mathrm{~nm}$, fluctuations in isolated grains could in fact destroy superconductivity [4]. The actual grain size distribution should thus be explored. The available TEM pictures were not of much help because there were many superposed layers of grains in the films, so that individual grains did not appear clearly. A different technique had to be used. The problem was solved in Tel Aviv thanks to a close collaboration with a talented expert in electron microscopy, the late Enrique Grunbaum, who suggested to use for this purpose dark-field electron microscopy where one can look only at grains that diffract in a certain direction. Individual grains then appeared very clearly. Results were spectacular. Grain size histograms were obtained for films having different oxide contents, controlled through the partial pressure of oxygen in the vacuum chamber and/or by the Al evaporation rate. At low oxide contents, histograms were very broad, grain sizes ranging from a couple of nm up to $10 \mathrm{~nm}$ or more [5]. The homogeneity of these films was therefore questionable. However, at high oxide content, histogram widths narrowed down, with grain sizes falling all below the critical value. This was most clearly the case for films grown on liquid nitrogen cooled substrates, where a very narrow size distribution around $2 \mathrm{~nm}$ was found [6]. The papers that resulted from this collaboration $[5,6]$ are still heavily quoted.

Back at Rutgers Peter Lindenfiled also got interested in granular superconductivity. Heat capacity measurements of granular 
films were performed in his group. They showed that films with a narrow grain size distribution, having normal state resistivity values of up to about 1000 micro-ohm-cm, did display a full BCS transition [7]. It occurred exactly at the same temperature as the resistive transition. Superconductivity in granular aluminum was clearly a bulk phenomenon, even at high resistivity values.

\subsection{The Percolation Argument of Ben Abeles: Could Josephson Be Wrong?}

Abeles assumed that inter-grain resistances due to oxide barriers dominated the macroscopic resistance of the films. In this model, the value of the inter-grain resistance is just the macroscopic resistivity divided by the grain size. Using BCS gap values Abeles calculated in this way that the Josephson coupling energy was by several orders of magnitude smaller than the electrostatic charging energy of the grains. He concluded that superconductivity in high resistivity granular films could not be a bulk phenomenon. It could only be a percolating phenomenon due to the presence of chains of grains with weak inter-grain resistances, something that could well happen due to disorder [8].

Yet the heat capacity measurements performed at Rutgers clearly showed otherwise. More recently, measurements of the optical conductivity of granular films have confirmed the presence of a clear gap edge and the bulk nature of superconductivity in high resistivity aluminum granular films [9]. These experiments clearly showed that the Abeles argument did not apply. What was wrong with it remained for many years an open question.

\subsection{The Role of Electron Localization: The Opposite Views of Dynes and Abeles}

Another but related problem concerned the value of the resistivity at which the metal to insulator transition occurs.

In two dimensions, Abrahams et al. [10] had predicted that weak disorder was sufficient to induce a non-metallic behavior. I had been much impressed by Elihu Abrahams during a short visit at Rutgers in 1980. I had taken literally their predictions that very weak disorder was sufficient to induce localization in 2D. While the groups at Bell Labs were trying to see the effect in strongly disordered thin films, I thought that if the predictions were correct, localization effects should be observable in simple thin copper films. These predictions were verified in a collaboration with the group of Bruynseraede in Louvain, where I was a guest for a few months in 1981, by very precise measurements on copper thin films. In these films, disorder is only due to non-specular electron reflections at the surfaces. In addition to the predicted logarithmic increase of the resistance of the films at low temperature, which could also be due to electron-electron interactions, we showed that these films displayed a negative magneto-resistance at fields of the order of a few hundred Gauss [11]. This was the first direct measurement of the localization length, and the first undisputable proof of 2D localization by weak disorder. Anderson himself was surprised by the success of their theory at this very weak disorder level. Contrary to what the Bell group had claimed, what they had observed was in fact interaction and not localization effects: the magneto-resistance of their highly disordered films was positive in strong fields, not negative at weak fields.

In $3 \mathrm{D}$, a stronger disorder is necessary to induce localization. According to the Ioffe Regel criterion, strong localization occurs when the scattering time $\tau$ is of the order of $\hbar / \mathrm{E}_{\mathrm{F}}$, where $\mathrm{E}_{\mathrm{F}}$ is the Fermi energy, or alternatively when $k_{F} l \approx l$, where $l$ is the mean free path. The Drude expression for the conductivity can be written as:

$\rho=\frac{\hbar}{e^{2} k_{F}} \frac{1}{k_{F} l}$

When $k_{F} l$ is of order unity, this expression gives the value of what is known as the Mott maximum metallic resistivity.

$\rho_{M}=\frac{\hbar}{e^{2} k_{F}}$

In fact, Mott had obtained this result in a different way [12], arguing that metallicity could not survive if the scattering rate became of the order of Planck's constant divided by the electron-electron Coulomb repulsion at interatomic distances, $e^{2} / a$. If $k_{F}$ is of the order of $a^{-1}$, or in other terms if there is one free electron per unit cell, the two results are the same.

It may be of interest to note that Abeles had obtained a similar result by considering the case of two grains weakly coupled together through a junction of resistance $R$ and capacitance $C$. He argued that electrons should be considered localized if the relaxation time of this $R C$ circuit, $\tau=R C$ was so large that electrons would "know" that they were isolated in the' individual grains:

$$
\frac{\hbar}{R C}<\frac{e^{2}}{2 C}
$$

or:

$R>\frac{2 \hbar}{e^{2}}$

where the number of nearest neighbors and other factors have not been taken into account. In the granular model, this translates into a maximum metallic resistivity:

$$
\rho=\frac{2 \hbar}{e^{2}} d
$$

where $d$ is the grain size. 
According to this argument for a grain size in the range of 2 to $3 \mathrm{~nm}$, the maximum metallic resistivity is of a few $100 \mu \Omega \mathrm{cm}$. This is similar to values obtained from the Yoffe-Regel or Mott criteria.

Beyond the Mott maximum metallic resistivity, the wave vector at the Fermi level is not a good quantum number, Cooper pairing should not work, superconductivity should be destroyed: the metal to insulator transition should coincide with the superconductor to insulator transition.

Dynes noted that beyond the Mott maximum metallic resistivity, the critical temperature of granular Al starts to decrease and interpreted this decrease as being in agreement with the localization argument. But this is disputable. In fact, beyond that resistivity, superconductivity should be destroyed, not just weakened. Localization theory cannot explain how 3D superconductivity can persist up to values of $k_{F} l$ as low as 0.01 , as observed by resistive measurements Abeles [8], Dynes [13], ourselves [5], and others, and by heat capacity [7] and optical [9] measurements.

In fact, superconductivity in granular aluminum persists up to resistivity values of the order of at least $30,000 \mu \Omega \mathrm{cm}$ as shown by the groups at Tel Aviv [5, 6], Bell Labs [13], Rutgers [14], and others. Localization theory cannot explain this experimental fact.

We know now that the localization argument works in fact very well when disorder is on the atomic scale. It successfully explains the destruction of superconductivity in $\mathrm{NbN}_{\mathrm{x}}$ where disorder is introduced by vacancies on the $\mathrm{N}$ sites [15]. Indeed, in this material, when $k_{F} l \approx l$, superconductivity is completely destroyed. But in granular aluminum, it is only destroyed when $k_{F} l \approx 0.01$. Some fundamental element is missing, likely related to granularity.

In summary, up to the 1980s research on grAl left open a major question: why can metallicity and superconductivity persist, as a bulk phenomenon, up to resistivity values at which $k_{F} l \ll 1$ ?

Two essential elements were missing to achieve a comprehensive understanding of granular superconductivity: the role of electron level splitting in the small grains and the exact nature of the metal to insulator transition.

\subsection{Electron Confinement and the Kondo Effect}

Two-nanometer size Al grains contain on the order of 1000 electrons. With the Fermi energy of $\mathrm{Al}$ being about $10 \mathrm{eV}$, the splitting between energy levels is roughly $10 \mathrm{meV}$, not much smaller than the electrostatic charging energy (estimated to be about $30 \mathrm{meV}$ ). It is only recently that the interplay of the splitting and charging energies has been studied, including also the role of inter-grain coupling [16].

First of all, this coupling reduces the charging energy, which explains why it can in fact be smaller than the Josephson coupling energy. But more fundamentally, the interplay between the energy level splitting and the (larger) Coulomb charging energy is not trivial. Conductance through a dot is dominated at high temperature by the Coulomb energy, but at temperatures below the level splitting, conductance can be restored by the Kondo resonance. In nano-scale granular materials, the energy scales are just right for this effect to be observable at temperatures that can be reached easily. In addition, Florens and Georges showed that when level broadening is sufficient for several levels to be involved, the Kondo effect can act twice. As a multi-level Kondo effect, it can induce a metallic behavior at higher temperatures, before the Coulomb energy reduces the conductance. At a still lower temperature, smaller than the level splitting, the single level Kondo resonance is again turned on. In the end, conductance through the dot is metallic at high temperature, then semiconducting, and metallic again at lower temperatures. This non-monotonous temperature dependence of the conductance has been observed experimentally in granular aluminum, see Fig. 1. It is made possible by the fact that the level splitting and the Coulomb energy are of the same order of magnitude, as discussed above [17]. Here, it is the Kondo resonance that plays a crucial role. Of course, the theory of Florens and Georges applies strictly only to conductance through a single dot, not to a 3D network of dots. But the non-trivial, nonmonotonous conductance behavior shown Fig. 1 is an encouraging indication that a similar effect is taking place in granular aluminum.

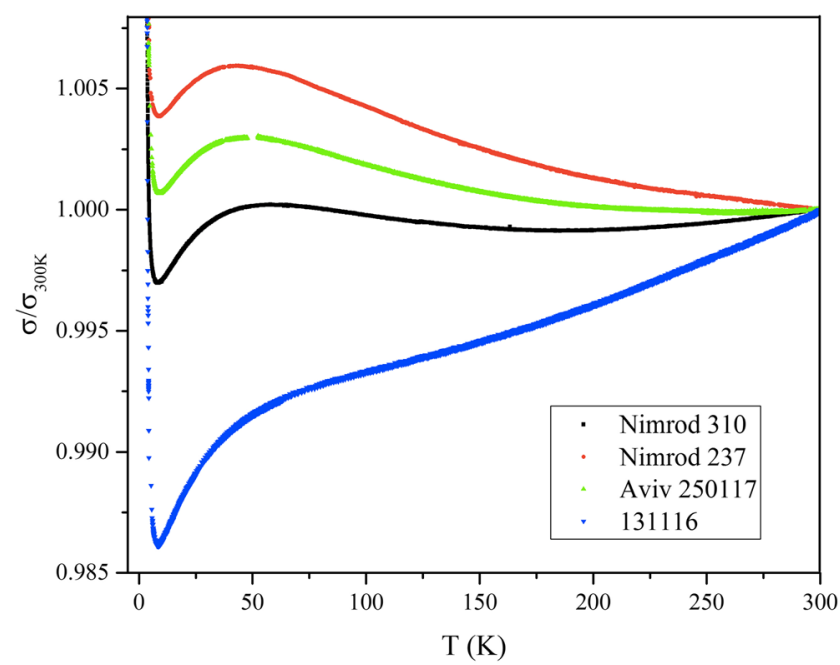

Fig. 1 Conductivity versus temperature for selected granular aluminum films, normalized to their room temperature value. The two upper curves are for samples with relatively well coupled grains (resistivity values of a few $100 \mu \Omega \mathrm{cm}$ ). The high-temperature metallic behavior is ascribed to a multi-level Kondo effect; a semiconducting behavior occurs when the temperature is of the order of the electrostatic energy. Finally, when the temperature falls below the level splitting, the single level Kondo effect restores a metallic behavior. The lowest curve is for weakly coupled grains. The Coulomb blockade dominates until the single level Kondo effect takes over (presented at the Superstripes 2017 meeting in Ischia, see ref. 17) 
Can the Kondo effect also enhance superconductivity? Choi et al. (M. S. Choi, M. Lee, K. Kang, and W. Belzig, Phys. Rev. B 70, 2 (2004)) have calculated the gap in a nano-dot coupled to superconducting banks. They found that it is in fact larger than that in the banks, suggesting that the Kondo effect in a granular metal can in fact enhance superconductivity.

\subsection{The Role Played by the Mott Transition}

Regarding the nature of the metal to insulator transition, Mott had shown that electron-electron repulsion could turn a metal into an insulator if the repulsive potential $U$ was larger than the bandwidth $D$ [12]. No disorder was involved in this transition. A gap would open up within the band width, between a lower and an upper Hubbard band. The consensus was that the density of states at the Fermi level would progressively decrease, until the Mott Hubbard gap would open up.

It turned out that things were actually more interesting. It was shown that, as Coulomb interactions are turned on, the density of states at the Fermi level remains in fact finite up to the transition [18]. A narrow band of itinerant states is formed around the Fermi level. It is the width of this band that vanishes at the transition, not the density of states. The effective mass of itinerant electrons goes to infinity at the transition.

It is very tempting to apply this approach to granular materials, with the grains electrostatic charging energy playing the role of the repulsive Coulomb potential and the energy level splitting that of the bandwidth. However, disorder is also present. Before going into the details, the first question to ask is whether one could combine the effects of disorder à la Anderson and Coulomb interaction à la Mott-HubbardGeorges?

\subsection{A Modified loffe Regel Criterion Near a Mott Metal to Insulator Transition}

This general problem is still pending. We propose here a heuristic argument to obtain the maximum metallic resistivity in the case where a disordered metal is near a Mott transition, itinerant electrons near the Fermi level having an effective mass $\mathrm{m}^{*}$.

We propose that the Ioffe Regel criterion should then be written in terms of the re-normalized Fermi energy:

$E_{F}^{*}=\frac{m}{m^{*}} E_{F}$

Assuming that the itinerant carriers are those in the narrow re-normalized band one gets for the maximum metallic resistivity in the vicinity of a Mott transition:

$\rho_{M M}=\frac{m^{*}}{m} \rho_{M}$
In this way, near a Mott transition, the persistence of metallicity and superconductivity up to resistivity values much larger than that predicted by the usual Ioffe-RegelMott criterion can be understood.

\subsection{BCS to Bose-Einstein Condensation Crossover}

Since the early work of Nozieres and Schmitt Rink on the existence of a continuous crossover between BCS and BoseEinstein condensations [19], there has been so far little contact between research on that crossover regime on the one hand, and superconductivity in the vicinity of a Mott transition on the other hand. However, if superconductivity persists up to a Mott M/I transition, it will necessarily be strong coupling as the effective narrowing bandwidth approaches the gap value. A signature of the vicinity of a Mott transition should therefore be strong coupling effects.

When the bandwidth and the superconducting gap become of the same order of magnitude (and so does by the way also the Kondo temperature), the number of Cooper pairs in a coherence volume becomes of order unity. This is a basic characteristic of a BCS to BE crossover. Strinati et al. [20] have studied how in this region the gap and the critical temperature vary in the vicinity of the unitary limit. They calculated how the strong coupling ratio varies as a function of $\left(k_{\mathrm{F}} a\right)^{-1}$, where $a$ is the scattering length that goes to infinity in the unitary limit. They found that $2 \Delta / k T_{c}$ goes from the BCS low coupling limit value 3.5 to 6 at the unitary limit.

Strong coupling effects were indeed reported by Moshe et al. [9] who measured recently the optical conductivity of grAl films up to near the M/I transition. The real and imaginary parts of the conductivity $\sigma(\omega)$ were fitted successfully to the Mathis Bardeen theory, with the gap edge clearly identified. The strong coupling ratio $2 \Delta / k T_{c}$ was found to increase in a systematic way from the BCS weak coupling value of 3.5 for low resistivity films, up to 4.5 for films having resistivity values of $5000 \mu \Omega \mathrm{cm}$. This work is still in progress, but it is already clear that strong coupling effects, consistent with a $\mathrm{BCS}$ to $\mathrm{BE}$ crossover region, show up in the vicinity of the transition.

The vicinity of a Mott transition in grAL can explain both experimental findings - the persistence of metallicity and superconductivity up to $k_{F} l$ values much smaller than unity, and the strong coupling effects observed in the vicinity of the transition. We emphasize that a Mott transition can only occur in granular materials if the grain size is so small that the energy level splitting in isolated grains approaches their electrostatic charging energy.

While much theoretical progress has been done recently in understanding the BCS to BE crossover region [20-22], a detailed link with theories of the Mott transition remains to be established. More experimental work is also necessary, for 
instance to identify the occurrence of strong fluctuation effects predicted to occur in that region.

Acknowledgments I would like to express my deep thanks to Philippe Nozières who through the years has guided me through the understanding of the BCS to Bose-Einstein condensation crossover regime, a regime whose very existence was not well accepted by Bardeen himself. I have also greatly benefited from his physical insight on recent developments regarding the behavior of the density of states near a Mott transition. On that topic, illuminating conversations with Antoine Georges are gratefully acknowledged. I have greatly benefited from continuing and fruitful exchanges with Giancarlo Strinati on strong coupling effects in the BCS to $\mathrm{BE}$ crossover, which have helped us in a decisive way to understand the significance of the optical conductivity experiments of Aviv Moshe and the earlier ones of Nimrod Bachar, whom I wish to thank here for our exciting collaboration. Many of the ideas presented here were discussed at the Superstripes meetings organized by Antonio Bianconi, whom I wish to thank warmly for his continuing interest in granular superconductivity.

\section{References}

1. Abeles, B., Cohen, R.W., Stowell, R.W.: Phys. Rev. Lett. 18, 902 (1967)

2. Cohen, R.W., Abeles, B.: Phys. Rev. 168, 444 (1967)

3. Parmenter, R.H.: Phys. Rev. 154, 353v (1967)

4. Deutscher, G.: Phys. Lett. A. 35, 28 (1971)

5. Deutscher, G., Fenichel, H., Gershenson, M., Grünbaum, E., Ovadyahu, Z.: J. Low Temp. Phys. 10, 231 (1973)

6. Deutscher, G., Gershenson, M., Grübaum, E., Imry, Y.: J. Vac. Sci. Technol. 10, 637 (1973)
7. Worthington, T., Lindenfeld, P., Deutscher, G.: Phys. Rev. Lett. 41, $316(1978)$

8. Abeles, B.: Phys. Rev. B. 15, 2828 (1977)

9. Moshe, A.G., Farber, E., Deutscher, G.: Phys. Rev. B. 99, 224503 (2019)

10. Abrahams, E., Anderson, P.W., Licciardello, D.C., Ramakrishnan, T.V.: Phys. Rev. Lett. 42, 673 (1979)

11. Van den dries, L., Van Haesendonck, C., Bruynseraede, Y., Deutscher, G.: Phys. Rev. Lett. 46, 565 (1981)

12. Mott, N.F.: Metal-insulator transitions. Taylor and Francis, London (1974)

13. Dynes, R.C., Garno, J.P.: Phys. Rev. Lett. 46, 137 (1981)

14. Kunchur, M., Lindenfeld, P., McLean, W.L., Brooks, J.S.: Phys. Rev. Lett. 59, 1232 (1987)

15. Cheng, B., Wu, L., Laurita, N.J., Singh, H., Chand, M., Raychaudhuri, P., Armitage, N.P.: Phys. Rev. B. 93, 180511(R) (2016)

16. Florens, S., San Jose, P., Guinea, F., Georges, A.: Phys. Rev. B. 68, 245311 (2003)

17. Moshe, A., Bachar, N., Lerer, S.: Yossi Lereah and Guy Deutscher. J. Supercond. Nov. Magn. 31(3), 733-736 (2018)

18. Georges, A., Kotliar, G., Krauth, W., Rozenberg, M.J.: Rev. Mod. Phys. 68, (1996)

19. Nozières, P., Schmitt-Rink, S.: J. Low Temp. Phys. 59, 195-211 (1985)

20. Palestini, F., Strinati, G.C.: Phys. Rev. B. 89, 224508 (2014)

21. Pisani, L., Perali, A., Pieri, P., Calvanese Strinati, G.: Phys. Rev. B. 97, 014528 (2018)

22. Pisani, L., Pieri, P., Calvanese Strinati, G.: Phys. Rev. B. 98, 104507 (2018)

Publisher's note Springer Nature remains neutral with regard to jurisdictional claims in published maps and institutional affiliations. 